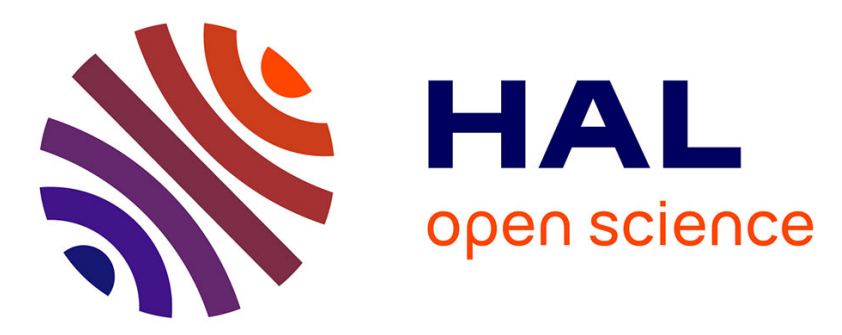

\title{
Static force transducer based on resonant piezoelectric structure: Root cause investigation
}

\author{
Salaheddine Safour, Yves Bernard
}

\section{To cite this version:}

Salaheddine Safour, Yves Bernard. Static force transducer based on resonant piezoelectric structure: Root cause investigation. Smart Materials and Structures, 2017, 26 (5), 10.1088/1361-665X/aa63da . hal-01705475

\section{HAL Id: hal-01705475 \\ https://hal.science/hal-01705475}

Submitted on 9 Feb 2018

HAL is a multi-disciplinary open access archive for the deposit and dissemination of scientific research documents, whether they are published or not. The documents may come from teaching and research institutions in France or abroad, or from public or private research centers.
L'archive ouverte pluridisciplinaire HAL, est destinée au dépôt et à la diffusion de documents scientifiques de niveau recherche, publiés ou non, émanant des établissements d'enseignement et de recherche français ou étrangers, des laboratoires publics ou privés. 


\title{
Static force transducer based on resonant piezoelectric structure: Root cause investigation
}

\author{
Salaheddine Safour and Yves Bernard \\ GeePs Laboratory, CENTRALESUPELEC-CNRS-UPSud-UPMC, Gif sur Yvette, France \\ E-mail: salaheddine.safour@gmail.com, yves.bernard@centralesupelec.fr
}

\begin{abstract}
The aim of this paper is to investigate the design and the integration of a static force sensor based on a resonant bulk acoustic wave piezoelectric transducer. Experimental measurements of the electrical admittance spectrum show its dependence on the applied force. We noticed that the quality of the frequency response for a given vibration mode degrades with the increase of the applied force, which limits the force measuring range. The root cause of the response-stress dependence is investigated by means of the developed analytical model and a finite element analysis. The analysis results show that the selected operating vibration mode, the components of the structure on which the piezoelectric specimen is positioned and the boundary conditions at the contact interfaces between all the components have a direct effect on the electrical admittance spectrum. A modified structure was proposed to enhance the quality of the frequency response. The experimental tests show higher measuring range (up to $1500 \mathrm{~N}$ ).
\end{abstract}

Keywords: force sensor, piezoelectric transducer, resonance, vibration mode, electrical admittance spectrum, analytical modeling, FEA

\section{Introduction}

Piezoelectric materials are reversible electromechanical converters; they can be found in nature as single crystal (e.g. Quartz) or synthesized as polycrystalline that are poled to acquire polarization at a macroscopic level (e.g. PZT). PZT ceramics have excellent piezoelectric properties, low costs and can easily take any complex shape [1]. Piezoelectric force sensors in the market are based on both material types; they are widely used due to their good linearity, high sensitivity, wide frequencyresponse range and simple integration $[2,3]$. The sensing techniques can be divided into two types: non-resonant and resonant (Figure. 1).

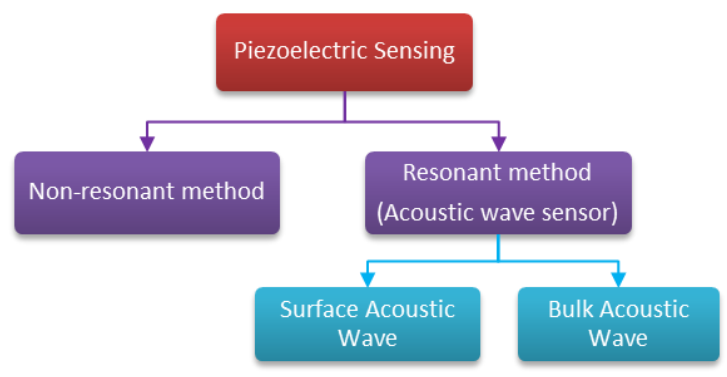

Figure. 1: Piezoelectric force sensing techniques

In non-resonant piezoelectric sensing, the direct effect combined with appropriate conditioning electronics (e.g. charge amplifier) is used [4]. While this sensing technique can be used for dynamic measurement it is not suitable for static measurement due to leakage on the material itself and the added electronics, therefore limiting the direct time constant (DTC) $[5,6,7]$. In order to sense the static component, methods based on the resonant-type sensor are more appropriate. This is based on the analysis of the material electromechanical response in time domain (ring-down) or the frequency domain (resonance frequency tracking) [8, 9, 10, 11]. The resonant piezoelectric sensors are usually designed to operate at or around their resonance frequency and can be divided into two types: surface acoustic wave sensors and bulk acoustic wave sensors. In the surface acoustic wave sensors (SAW), the vibration wave propagates on the surface of the material $[12,13,14]$. This sensor is based on a substrate or diaphragm placed between two piezoelectric elements, the first one uses the inverse effect and the second one the direct effect. The pressure applied to the diaphragm (substrate) leads to its deformation, consequently changing the propagation delay; therefore, the pressure could be calculated based on the propagation delay measurement. On the other hand, in the bulk acoustic wave sensors (BAW) the vibration wave propagates on the piezoelectric material volume which is often implemented in the form of a micro electromechanical system (MEMS) that is in contact with fluid.

The targeted application of most literature on this subject is pressure measurement $[15,16,17,18]$, for very lowforce sensing only a few works focus on high level physical mechanisms $[19,20]$, while other authors focus more on the design of the signal conditioning electronics 
that use the resonance frequency shift without any attempt to analyze or explain the phenomenon observed on the response of the resonant transducer[21, 22]. The novelty of the work presented in this paper is the utilization of a bulk ring-shaped piezoelectric specimen (required by the application) positioned within a mechanical structure to attempt to measure traction and compression static force by means of the resonance operating mode. Figure. 2 shows part of the mechanical structure under consideration for the application. The goal is to provide guidance based on the best understanding of the physical mechanisms in order to allow optimal design of the transducer, which is capable of ensuring the purity of the response when applying high uniaxial static force. The salient point of the electrical admittance spectrum is selected to act as the direct measurement of the applied force. The feasibility investigation of such a transducer in this work is based on analytical modeling, finite element analysis and experimental measurements.

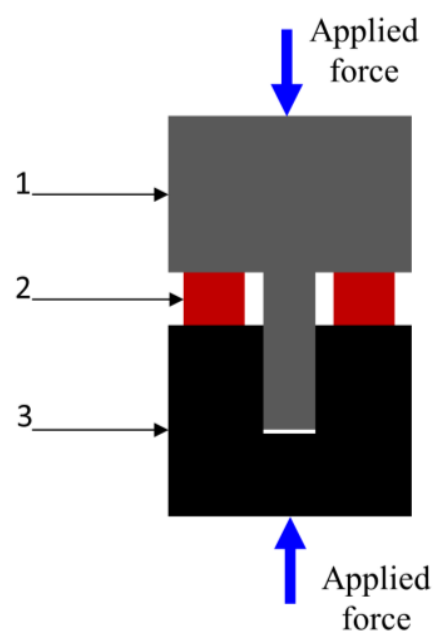

Figure. 2: Cross section of a part of the studied structure $\{(1)$ cylindrical bar with screw, (2) ring shaped piezoelectric material, (3) cylindrical bar with taped hole\}.

\section{Stress effect on the response of the piezoelectric specimen}

In this section the effect of the applied mechanical stress on the electrical admittance of a piezoelectric specimen is shown by means of experimental measurement. Prior to the analysis of the stress effect, we started with the identification of the free vibration modes of a ringshaped piezoelectric specimen based on PZT material NCE 41 from Noliac (dimensions: OD $35 \mathrm{~mm}$, ID 15 $\mathrm{mm}$, TH $6.35 \mathrm{~mm}$ ) in order, later on, to have an idea of the interaction between the specimen and the mechanical structure.

\subsection{Free vibration modes identification}

The free vibration modes of the piezoelectric specimen were analyzed through electrical admittance spectrum measurement. Figure. 3 shows the experimental setup based on an impedance analyzer (Agilent 4294a) with low AC voltage amplitude $\left(\mathrm{V}_{\mathrm{p}}=0.5 \mathrm{~V}\right)$.

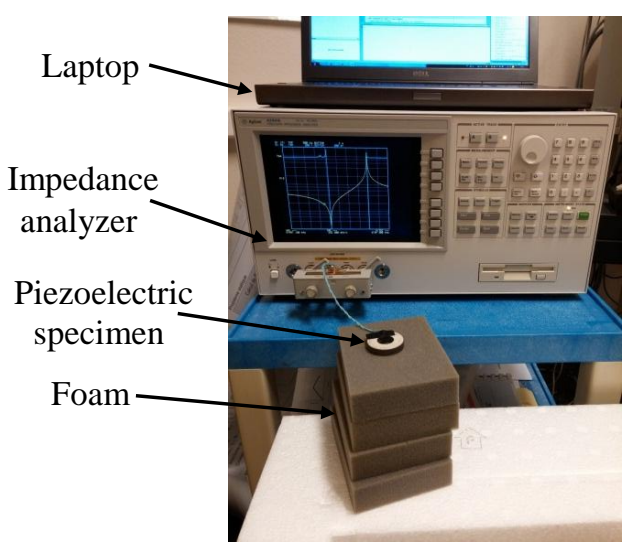

Figure. 3: Experimental setup of the electrical admittance measurement of the free piezoelectric specimen

Figure. 4 shows the existence of two vibration modes in the frequency range of $30 \mathrm{kHz}-200 \mathrm{kHz}$, each of them being identified by the local maximum of the admittance spectrum modulus and the phase angle switching from $90^{\circ}$ to $-90^{\circ}$. The quality factor of the first mode is around 250 and that of the second mode is around 1000. It can also be seen that the second mode has a higher electromechanical coupling than the first mode which is characterized by the distance between the resonance frequency and the anti-resonance frequency. Both vibration modes will be used in the stress effect analysis section.
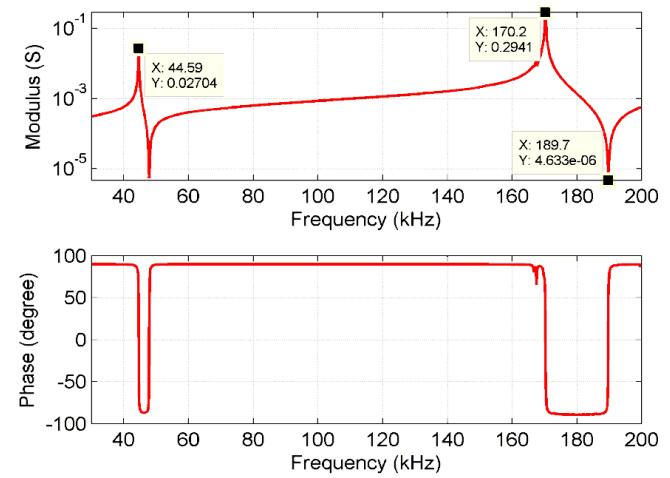

Figure. 4: Measured electrical admittance spectrum of the free piezoelectric specimen

The mechanical displacement was measured using a laser Doppler vibrometer (Polytec PSV-I-500). The piezoelectric specimen was excited by applying a periodic chirp signal that consists of a superposition of sinusoidal signals, which allows the excitation of all the natural frequencies within the desired frequency band. 
Figure. 5 shows a significant spike in the mechanical displacement that characterizes a resonance frequency at $44.46 \mathrm{kHz}$ which is in good agreement with the resonance frequency of the first vibration mode obtained by the admittance measurement. The figure also shows the presence of multiple resonance modes in the frequency range $10 \mathrm{kHz}$ to $100 \mathrm{kHz}(100 \mathrm{kHz}$ is the maximum frequency allowed by the instrument) that are not measurable with the impedance analyzer.

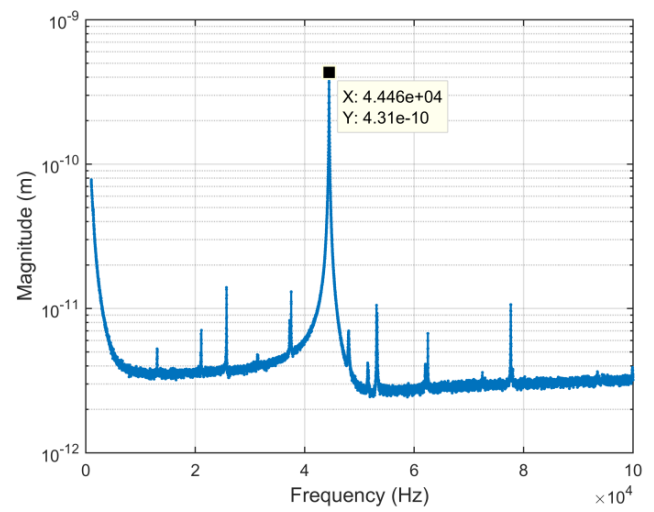

Figure. 5: Measured frequency spectrum of the mechanical displacement as function of the frequency

A finite element method based tool (COMSOL Multiphysics) was used in order to have an idea of the shape of the vibration modes. The electrical admittance spectrum of the free vibration piezoelectric specimen is in agreement with the measurement (Figure. 6.a). The first mode is a radial vibration mode according to its deformation shape, while the second one has a more complex shape with non-neglected thickness displacement.
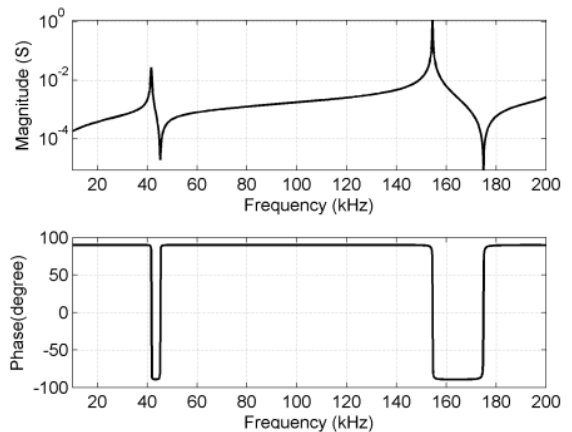

(a)

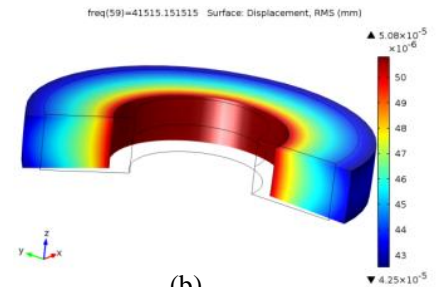

(b)

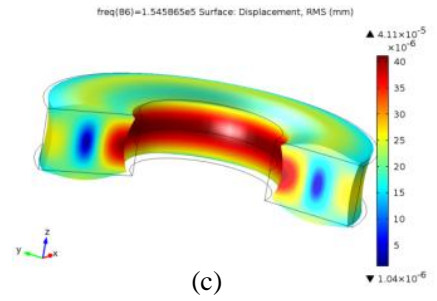

(c)
The eigenfrequency analysis shows the presence of more than two modes in the frequency range of $10 \mathrm{kHz}$ to 200 $\mathrm{kHz}$, which matches the interferometry measurement results. Those modes neither appear in the frequency domain simulation or in the electrical admittance measurements; this might be due to the fact that those modes have very low electromechanical coupling and hence require very high energy in order to be detectable by the measuring instrument.

\subsection{Observation of the stress effect on the resonant piezoelectric structure}

The intent of this section is to analyze the sensitivity of the frequency response of the piezoelectric specimen to the applied static force. The specimen was positioned between two steel bases of the Zwick/Roell Z030 compression machine (Figure. 7); the utilized auxiliary electrodes, which were based on aluminum foil (thickness $=0.1 \mathrm{~mm}$ ) with insulated paper (thickness = $0.1 \mathrm{~mm}$ ) on one face were used to excite the specimen with an AC voltage applied in the poling direction (the force was applied in the same direction).

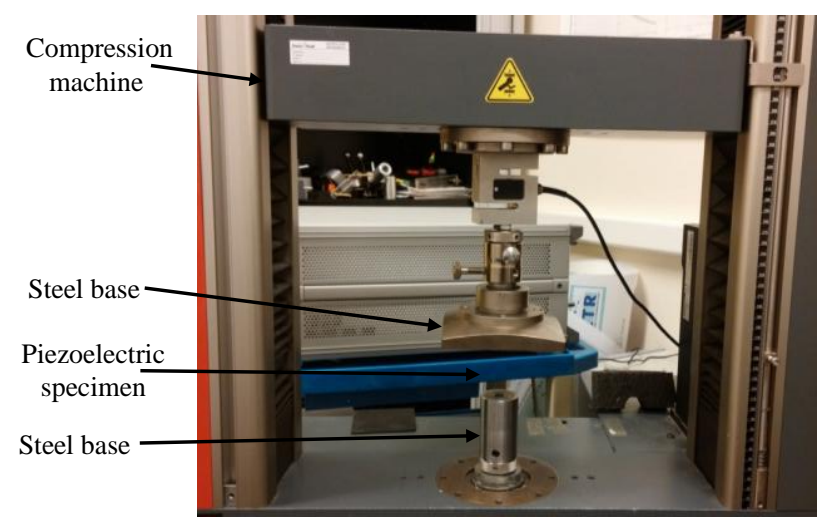

Figure. 7 : Experimental setup for stress effect investigation

Figure. 8 shows the variation of the magnitude and phase of the electrical admittance for the first and second vibration modes with the applied force (maximum/minimum admittance magnitude variation, zero phase angle point shifting). However, the resonance frequencies remain close to those obtained in free vibration condition.

Figure. 6: FE simulation results (a) admittance spectrum (b) first vibration mode shape (c) second vibration mode shape 

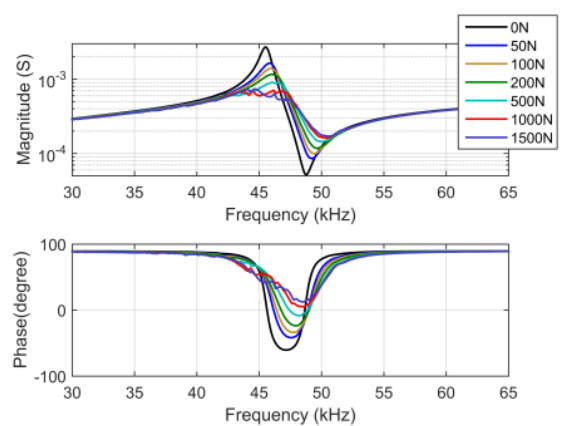

(a)
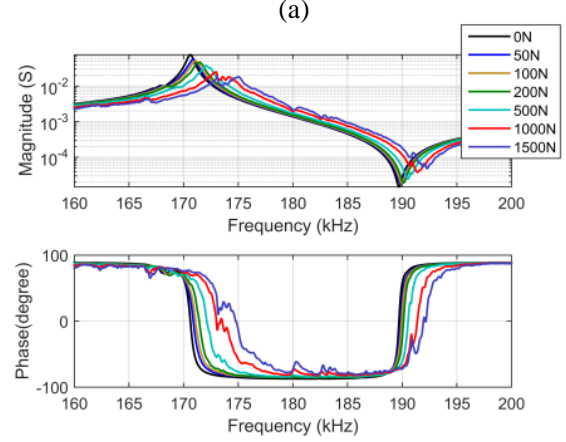

(b)

Figure. 8: Measured electrical admittance for (a) the first vibration mode and (b) the second vibration mode

Figure 9.a and $\mathrm{b}$ show that the resonance frequency variation for both modes is not linear with respect to the applied force. The maximum sensitivity for the first mode is $10 \mathrm{~Hz} / \mathrm{N}$ while it remains around $4.55 \mathrm{~Hz} / \mathrm{N}$ for the second mode; the plotted force range was reduced to $500 \mathrm{~N}$ due to the unusable curves for higher force values. The quality factor drops dramatically when the specimen is inserted within the structure (from 250 to 28 for the first mode and from 1000 to 162 for the second mode).

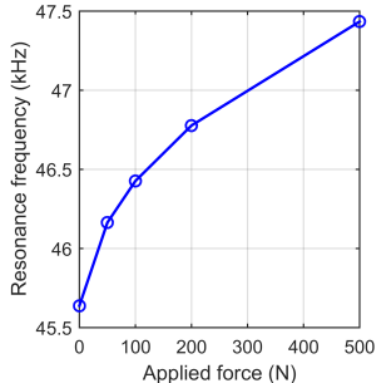

(a)

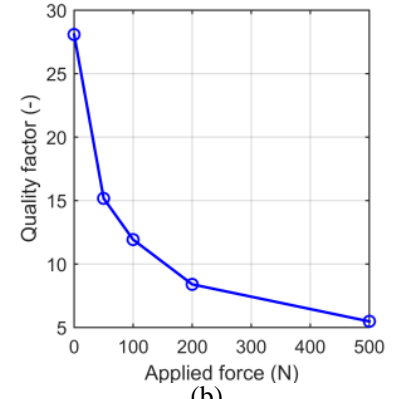

(b)

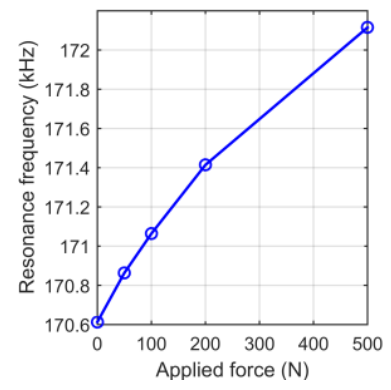

(c)

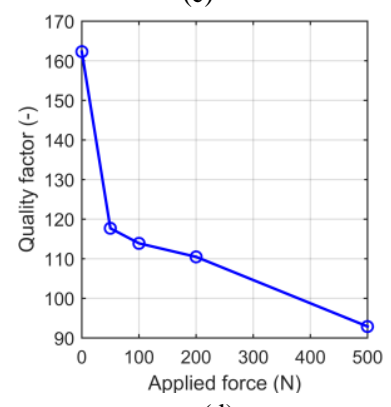

(d)
Figure. 9: Resonance frequency and quality factor variation for $(a, b)$ the first mode and (c,d) the second mode as a function of the applied static force

An applied force higher than $500 \mathrm{~N}$ leads to unusable spectrum for the first mode, while the spectrum of the second mode shows the appearance of unwanted parasitic modes which show small variations in the magnitude and phase. The amplitude of those parasitic modes increases as the stress in increased. The effect of the added insulated paper on the parasitic modes is considered insignificant in this study due to its thinness $(0.1 \mathrm{~mm})$.

Note that the second vibration mode has higher electromechanical coupling than the first one, a characteristic that could allow for a wide measuring range if the parasitic modes had been eliminated.

\section{Stress effect root cause investigation}

In order to achieve an effective design of the force transducer with high sensitivity and measuring range, we need to understand the root cause of: the shift of the resonance and anti-resonance frequencies, the qualityfactor variation and the parasitic modes that degrade the quality of the electrical admittance spectrum. Four assumptions are put forward regarding the physical mechanisms that could impact the electrical admittance when the piezoelectric specimen is positioned within a structure and subjected to external stress:

- The variation of the properties of the piezoelectric material with respect to the applied force.

- The variation of energy losses

- The variation of the boundary condition at the contact interface between the components of the structure due to the surfaces roughness. The increase of the applied force enhances the acoustic coupling between the piezoelectric specimen and the rest of the structure.

- Large deformation

An analytical model was developed and used to analyze the effect of the first and second assumptions on the frequency response using the first vibration mode, while a finite element method-based model is used to investigate the third and fourth assumptions using the second vibration mode. The simulation result (using the non-linear geometry) regarding the last assumption shows that within the studied force range, the structure remains in the small deformation domain and without any effect on the electrical admittance.

\subsection{Effect of the material parameters variation on the frequency response}

The material properties of the piezoelectric material are stress-dependent according to Q. Zhang and J. Zhao 
[23]. In order to analyze the effect of the variation of the compliance coefficients, the piezoelectric coefficients and the electric coefficients on the frequency response of a ring-shaped specimen (Figure. 10), a linear analytical model that provides the electrical admittance spectrum for free vibration condition was developed.

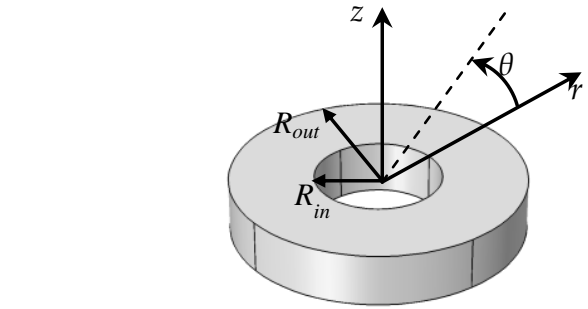

Where $R_{i}$ is the inner diameter, $R_{o}$ is the outer diameter

Figure. 10: Configuration of the ring shaped specimen

The model only takes into account the radial axisymmetric vibration modes. The following assumptions were made:

- Very small thickness (the out plane vibration and the two in-plane vibration are decoupled)

- The rectilinear element normal to the middle surface remains perpendicular after deformation, $e_{r z}=e_{\theta z}=0$

- Shear stresses are neglected

- The normal stress $\sigma_{z z}$ is very small and can be neglected, $\sigma_{z z}=0$

The equations of the radial motion in cylindrical coordinates and the strain-mechanical displacement relations in cylindrical coordinates are given by [24]:

$\frac{\partial \sigma_{r r}}{\partial r}+\frac{1}{r}\left(\sigma_{r r}-\sigma_{\theta \theta}\right)=\rho \frac{\partial^{2} u_{r}}{\partial t^{2}}$

$e_{r r}=\frac{\partial u_{r}}{\partial r}, e_{\theta \theta}=\frac{u_{r}}{r}$

The piezoelectric material used in this study is polarized across the thickness; consequently the charge equation (Maxwell-Gauss equation) and the electric field electrical potential equations are given by:

$\frac{\partial D_{z}}{\partial z}=0, E_{z}=\frac{-\partial \varphi}{\partial z}$

The piezoelectric constitutive equations are as follows:

$$
\begin{aligned}
& \sigma_{r r}=\frac{1}{s_{1 I}^{E}\left(1-v_{p}^{2}\right)}\left(e_{r r}+v_{p} e_{\theta \theta}\right)-\frac{d_{31}}{s_{1 I}^{E}\left(1-v_{p}\right)} E_{z} \\
& \sigma_{\theta \theta}=\frac{1}{s_{11}^{E}\left(1-v_{p}^{2}\right)}\left(v_{p} e_{r r}+e_{\theta \theta}\right)-\frac{d_{31}}{s_{11}^{E}\left(1-v_{p}\right)} E_{z} \\
& D_{z}=d_{31}\left(\sigma_{r r}+\sigma_{\theta \theta}\right)+\varepsilon_{33}^{T} E_{z}
\end{aligned}
$$

Where $v_{p}=\frac{-s_{12}^{E}}{s_{11}^{E}}$ is the planar Poisson's ratio.

For harmonic excitation and using the equations (2), (5) and (6) the equation of motion (1) can be expressed as:

$$
\frac{\partial^{2} U}{\partial r^{2}}+\frac{1}{r} \frac{\partial U}{\partial r}-\frac{U}{r^{2}}+k^{2} U=0
$$

Where $k^{2}=s_{11}^{E} \rho \omega^{2}\left(1-v_{p}^{2}\right)$ is the wave number.

The general solution of (9) is:

$$
U(r)=A J_{I}(k r)+B Y_{l}(k r)
$$

Where $J_{l}$ and $Y_{l}$ are Bessel functions of the first and second kind respectively.

From the boundary conditions, we have [25]:

$$
\left.\sigma_{r r}\right|_{r=R_{\text {in }}}=0,\left.\quad \sigma_{r r}\right|_{r=R_{\text {out }}}=0,\left.\varphi\right|_{z=h / 2}=V,\left.\quad \varphi\right|_{z=-h / 2}=0
$$

Using the boundary conditions, equations (5) and (10) lead us to determine the constants $A$ and $B$ of the general solution of (9):

$$
\begin{aligned}
A= & {\left[-2 d_{3 l}\left(v_{p}+1\right)\left(\alpha Y_{I}\left(k R_{\text {out }}\right) v_{p}-Y_{I}\left(k R_{\text {in }}\right) v_{p}-\alpha Y_{I}\left(k R_{\text {out }}\right)\right.\right.} \\
& \left.\left.+Y_{I}\left(k R_{\text {in }}\right)+\alpha \eta Y_{0}\left(k R_{\text {out }}\right)-\alpha \eta Y_{0}\left(k R_{\text {in }}\right)\right) R_{\text {out }} V\right] / h P_{I} \\
B= & {\left[2 d _ { 3 I } ( v _ { p } + 1 ) \left(\alpha J_{I}\left(k R_{\text {out }}\right) v_{p}-J_{I}\left(k R_{\text {in }}\right) v_{p}-\alpha J_{I}\left(k R_{\text {out }}\right)\right.\right.} \\
& \left.\left.+J_{I}\left(k R_{\text {in }}\right)+\alpha \eta J_{0}\left(k R_{\text {out }}\right)-\alpha \eta J_{0}\left(k R_{\text {in }}\right)\right) R_{\text {out }} V\right] / h P_{I}
\end{aligned}
$$

Where:

$$
\begin{aligned}
& P_{I}=\left[J_{I}\left(k R_{\text {in }}\right) v_{p}^{2} Y_{I}\left(k R_{\text {out }}\right)-2 J_{I}\left(k R_{\text {in }}\right) v_{p} Y_{I}\left(k R_{\text {out }}\right)\right. \\
& +\alpha \eta J_{0}\left(k R_{\text {in }}\right) v_{p} Y_{l}\left(k R_{\text {out }}\right)+J_{I}\left(k R_{\text {in }}\right) Y_{I}\left(k R_{\text {out }}\right) \\
& -\alpha \eta J_{0}\left(k R_{\text {in }}\right) Y_{l}\left(k R_{\text {out }}\right)-J_{I}\left(k R_{\text {out }}\right) v_{p}^{2} Y_{I}\left(k R_{\text {in }}\right) \\
& +2 J_{I}\left(k R_{\text {out }}\right) v_{p} Y_{I}\left(k R_{\text {in }}\right)-\eta J_{0}\left(k R_{\text {out }}\right) v_{p} Y_{l}\left(k R_{\text {in }}\right) \\
& J_{I}\left(k R_{\text {out }}\right) Y_{l}\left(k R_{\text {in }}\right)+\eta J_{0}\left(k R_{\text {out }}\right) Y_{l}\left(k R_{\text {in }}\right) \\
& +\eta J_{I}\left(k R_{\text {in }}\right) v_{p} Y_{0}\left(k R_{\text {out }}\right)-\eta J_{I}\left(k R_{\text {in }}\right) Y_{0}\left(k R_{\text {out }}\right) \\
& +\alpha \eta^{2} J_{0}\left(k R_{\text {in }}\right) Y_{0}\left(k R_{\text {out }}\right)-\alpha \eta J_{I}\left(k R_{\text {out }}\right) v_{p} Y_{0}\left(k R_{\text {in }}\right) \\
& +\alpha \eta J_{I}\left(k R_{\text {out }}\right) Y_{0}\left(k R_{\text {in }}\right)-\alpha \eta^{2} J_{0}\left(k R_{\text {out }}\right) Y_{0}\left(k R_{\text {in }}\right)
\end{aligned}
$$

With

$$
\alpha=\frac{R_{\text {in }}}{R_{\text {out }}} \text { and } \eta=k R_{\text {out }}
$$

The electrical current can be expressed as:

$$
\begin{aligned}
& I=i \omega \iint D_{z} d s \\
& =i \omega \int_{0}^{2 \pi R_{\text {out }}} \int_{R_{\text {in }}}\left[\frac{d_{31}}{s_{1 I}^{E}\left(1-v_{p}\right)}\left(\frac{\partial U}{\partial r}+\frac{U}{r}\right)+\frac{\varepsilon_{33}^{T} V}{h}\left(k_{p}^{2}-1\right)\right] r d r d \theta
\end{aligned}
$$


Where $k_{p}=\sqrt{\frac{2 d_{31}^{2}}{\varepsilon_{33}^{T} s_{11}^{E}\left(1-v_{p}\right)}}$

The electrical admittance is then given by:

$$
Y=i \omega \frac{2 \pi \varepsilon_{33}^{T} R_{\text {out }}^{2}}{h}\left[\left(1+v_{p}\right) k_{p}^{2} \frac{P_{2}}{P_{1}}+\left(k_{p}^{2}-1\right)\left(1-\alpha^{2}\right)\right]
$$

And

$$
\begin{aligned}
& P_{2}=\left[\alpha^{2} J_{I}\left(k R_{\text {in }}\right) v_{p} Y_{I}\left(k R_{\text {out }}\right)-J_{I}\left(k R_{\text {in }}\right) v_{p} Y_{I}\left(k R_{\text {out }}\right)\right. \\
& -\alpha^{2} J_{l}\left(k R_{\text {in }}\right) Y_{l}\left(k R_{\text {out }}\right)+J_{l}\left(k R_{\text {in }}\right) Y_{l}\left(k R_{\text {out }}\right) \\
& +\alpha \eta J_{0}\left(k R_{\text {out }}\right) Y_{1}\left(k R_{\text {out }}\right)-\alpha \eta J_{0}\left(k R_{\text {in }}\right) Y_{l}\left(k R_{\text {out }}\right) \\
& -\alpha^{2} J_{l}\left(k R_{\text {out }}\right) v p Y_{l}\left(k R_{\text {in }}\right)+J_{l}\left(k R_{\text {out }}\right) v_{p} Y_{l}\left(k R_{\text {in }}\right) \\
& +\alpha^{2} J_{l}\left(k R_{\text {out }}\right) Y_{l}\left(k R_{\text {in }}\right)-J_{l}\left(k R_{\text {out }}\right) Y_{l}\left(k R_{\text {in }}\right) \\
& -\alpha^{2} \eta J_{0}\left(k R_{\text {out }}\right) Y_{1}\left(k R_{\text {in }}\right)+\alpha^{2} \eta J_{0}\left(k R_{\text {in }}\right) Y_{I}\left(k R_{\text {in }}\right) \\
& -\alpha \eta J_{l}\left(k R_{\text {out }}\right) Y_{0}\left(k R_{\text {out }}\right)+\alpha^{2} \eta J_{l}\left(k R_{\text {in }}\right) Y_{0}\left(k R_{\text {out }}\right) \\
& +\alpha \eta J_{I}\left(k R_{\text {out }}\right) Y_{0}\left(k R_{i}\right)-\alpha^{2} \eta J_{I}\left(k R_{\text {in }}\right) Y_{0}\left(k R_{\text {in }}\right)
\end{aligned}
$$

From (15), the frequencies that correspond to the maximum and minimum of the modulus can be determined from the equations:

$$
P_{1}=0
$$

And

$$
\left(1+v_{p}\right) k_{p}^{2} P_{2}+\left(k_{p}^{2}-1\right)\left(1-\alpha^{2}\right) P_{1}=0
$$

The frequency of the maximum admittance is expressed as:

$$
f=\frac{k}{2 \pi} \sqrt{\frac{1}{\rho s_{11}^{E}\left(1-v_{p}^{2}\right)}}
$$

The initial values of the material properties were obtained from the material manufacturer's datasheet; the real material properties of our specimen were obtained by correlating the admittance spectrum obtained by the model with the measurements by using a logarithmic least-squared error algorithm.
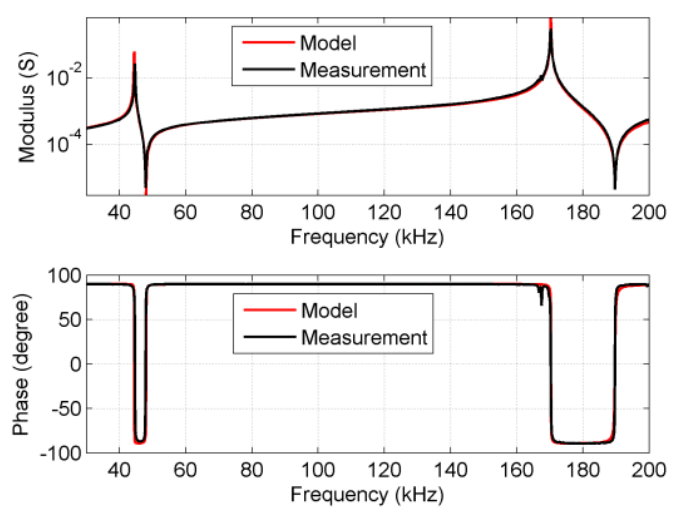

Figure. 11: Measured and simulated electrical admittance spectrum as a function of the frequency

Table 1. The parameters of the piezoelectric material.

\begin{tabular}{lll}
\hline & Manufacture & Identification \\
\hline$s_{11}^{E}\left[1 \mathrm{e}^{-12} \mathrm{~m}^{2} / \mathrm{N}\right]$ & $13( \pm 5 \%)$ & 12,56 \\
$s_{12}^{E}\left[1 \mathrm{e}^{-12} \mathrm{~m}^{2} / \mathrm{N}\right]$ & $\mathrm{NC}$ & $-4,43$ \\
$\mathrm{~d}_{31}\left[1 \mathrm{e}^{-12} \mathrm{C} / \mathrm{N}\right]$ & $-130( \pm 5 \%)$ & $-132,12$ \\
$\rho \quad\left[1 \mathrm{e}^{-12} \mathrm{Kg} / \mathrm{m}^{3}\right]$ & $7,9( \pm 5 \%)$ & 7,85 \\
$\mathrm{k}_{\mathrm{p}}[-]$ & $0,57( \pm 5 \%)$ & 0,599 \\
\hline
\end{tabular}

The result of the identification is shown in Figure. 11 and the obtained parameters are listed in Table 1. The simulation result shows the existence of two vibration modes as observed in the measurement, and referring to the model assumptions these modes are axisymmetric. Once the analytical model was validated, we used it to analyze the effect of the variation of different material parameters on the electrical admittance spectrum. The variations of the material properties were based on the data in the paper by Q. Zhang and J. Zhao [23] assuming that the parameters have the same linear variation with respect to the applied force (F) (e.g equation (20) for the compliance coefficient).

$s_{11}^{E}=s_{11 \_0}^{E}\left(1+10^{-5} \mathrm{~F}\right)$

Where $s_{11 \_0}^{E}$ is the compliance coefficient at zero applied force.

The simulation results in Figure. 12 show that the resonance frequency and the anti-resonance frequency are highly sensitive to the variation of the compliance coefficient $s_{11}^{E}$, and less sensitive to the other properties. 


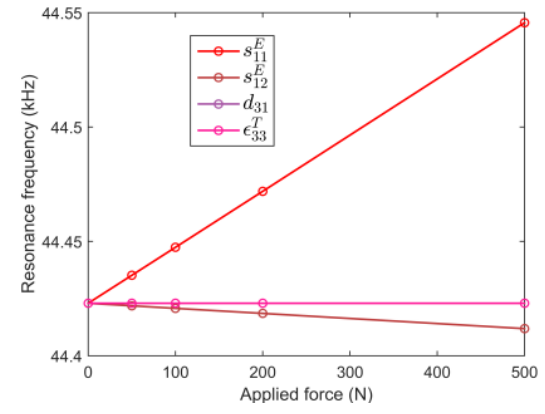

(a)

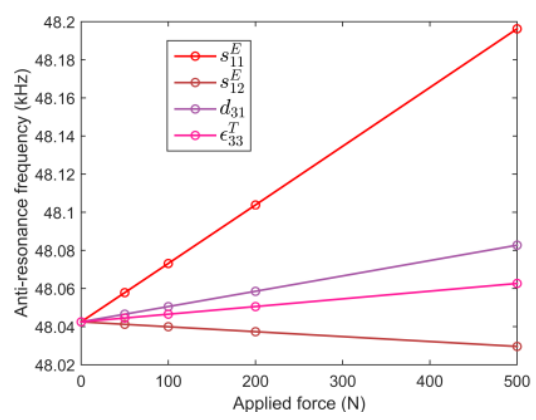

(b)

Figure. 12: The effect of the material properties variation on the (a) resonance frequency and (b) anti-resonance frequency of the first mode as a function of the applied force

The second point we investigated is the effect of both dielectric and mechanical losses by introducing complex permittivity and a compliance coefficient (elastic losses) to the model. The simulation results show that the effect of the dielectric losses is observed only at the antiresonance frequency; however the elastic losses modify the admittance spectrum at both the resonance and the anti-resonance frequencies (Figure. 13).

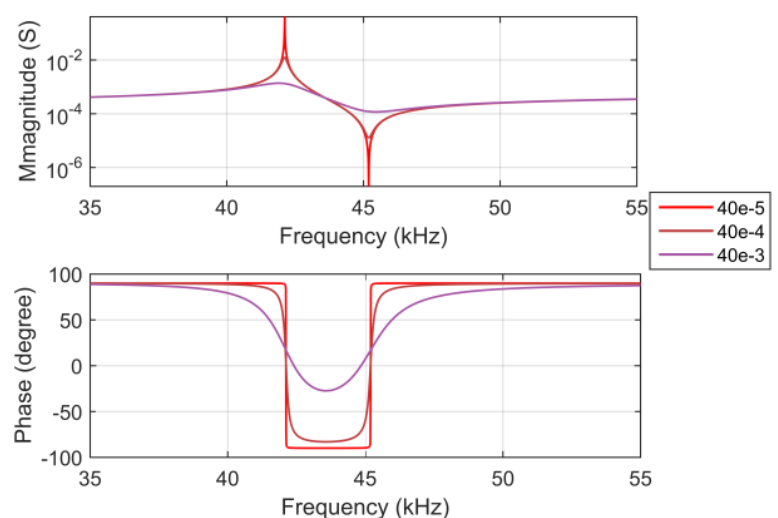

Figure. 13: Electrical admittance spectrum obtained for different values of the elastic loss factor

The combination of a linear variation of the material compliance coefficient $(10 \% / \mathrm{kN})$, the piezoelectric coefficient $(5 \% / \mathrm{kN})$ and the elastic losses $(1500 \% / \mathrm{kN})$ allows the replication of the effect of the applied force on the frequency spectrum for the first vibration mode with the following set of equations:

$$
\begin{aligned}
& s_{11}^{E}=s_{11 \_0}^{E}\left(1-10.10^{5} \mathrm{~F}\right) ; \quad s_{11 \_0}^{E}=12.56 .10^{12}\left[\mathrm{~m}^{2} / \mathrm{N}\right] \\
& d_{31}=d_{31 \_0}\left(1-5.10^{-5} \mathrm{~F}\right) ; \quad d_{31 \_0}=-13212.10^{-12}[\mathrm{C} / \mathrm{N}] \\
& \eta_{\text {mec }}=\eta_{\text {mec } 00}\left(1+15.10^{3} \mathrm{~F}\right) ; \quad \eta_{\text {mec } \_0}=80.10^{-4}[-]
\end{aligned}
$$

According to the numerical results shown in Figure. 14 the observed effect of the applied stress on the electrical admittance spectrum is due to a combination of the variation of the piezoelectric material's properties and of the increase of mechanical losses. The losses could be due to mechanical friction at the contact interfaces between the components of the structure or the propagation of the acoustic wave through the components. In our analytical model, all the potential mechanical losses of the structure were reduced to the piezoelectric material.
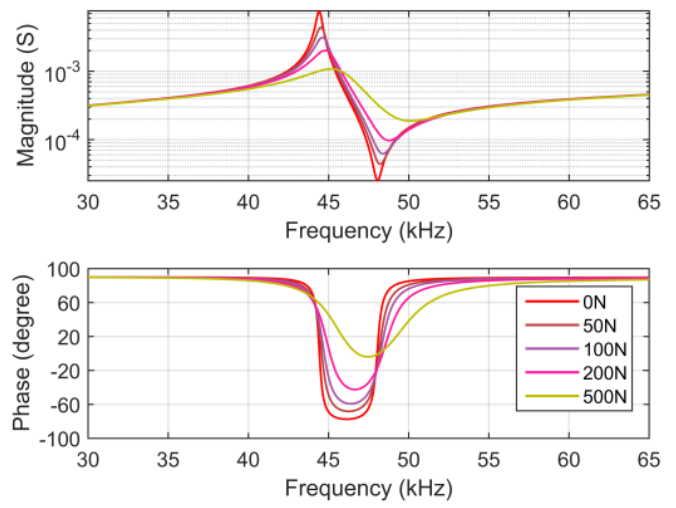

Figure. 14: Effect of the compliance coefficient and mechanical losses variation on the electrical admittance spectrum

\subsection{Effect of the mechanical boundary conditions on the frequency response}

In this section we investigate the contribution of the mechanical boundary conditions on the variation of the electrical admittance spectrum using a finite elementbased software (Comsol Multiphysics). This was achieved by taking into account the effect of the structure around the piezoelectric material. Since the structure is composed of different layers made of different materials, part of the acoustic wave generated by the piezoelectric specimen will be reflected due to the discontinuity of the acoustic impedance at the contact interfaces. First we analyzed the effect of the steel bases (Height $=100 \mathrm{~mm}$, side length $=60 \mathrm{~mm})$ on the electrical admittance spectrum assuming a perfect coupling (the continuity of the displacement field at the contact interface is ensured) between the piezoelectric specimen and the steel bases (Figure. 15). The second vibration mode was used in this study for its potential high-force measuring range. 


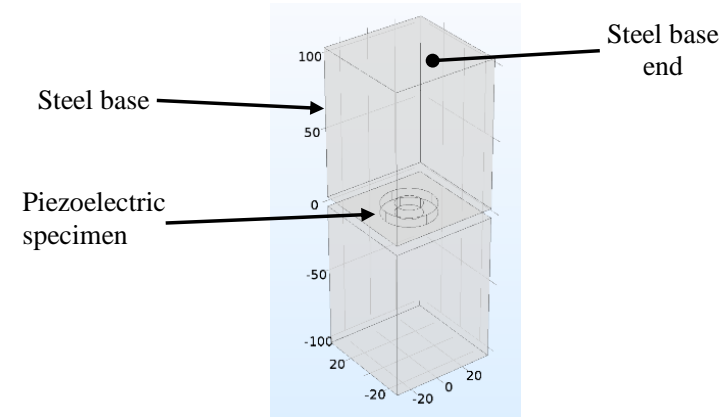

Figure. 15: Analyzed mechanical structure with the piezoelectric specimen (the steel bases height is equal to100 $\mathrm{mm}$ )

Figure. 16 clearly shows that the boundary condition at the interface between the piezoelectric specimen and the steel bases has an important effect on the electrical admittance. This also highlights the fact that a highly constrained displacement of the piezoelectric specimen degrades the purity of the admittance spectrum through the development of multiple unwanted vibration modes despite the disappearance of the main vibration mode. Therefore, in sensor application there is no interest in ensuring a perfect coupling between the piezoelectric specimen and the structure in which it will be positioned.
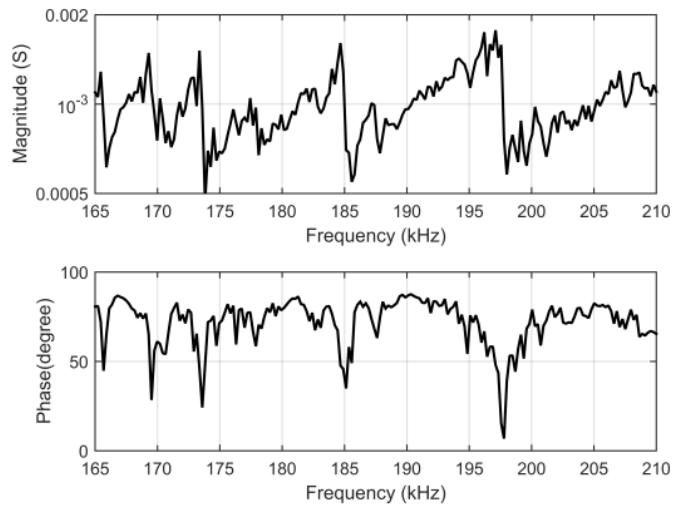

Figure. 16: Electric admittance spectrum of the piezoelectric specimen with the continuity of displacement boundary condition.

In the actual experimental structure, the piezoelectric material was not glued or soldered onto the steel bases; therefore, the continuity of the displacement boundary condition at the contact interface in the model is not to be considered. In order to approach the real boundary condition at the contact interface, a thin elastic layer with an equivalent stiffness was added between the piezoelectric specimen and the structure to model the used auxiliary electrodes with the insulation paper under the assumption of not sliding contact.
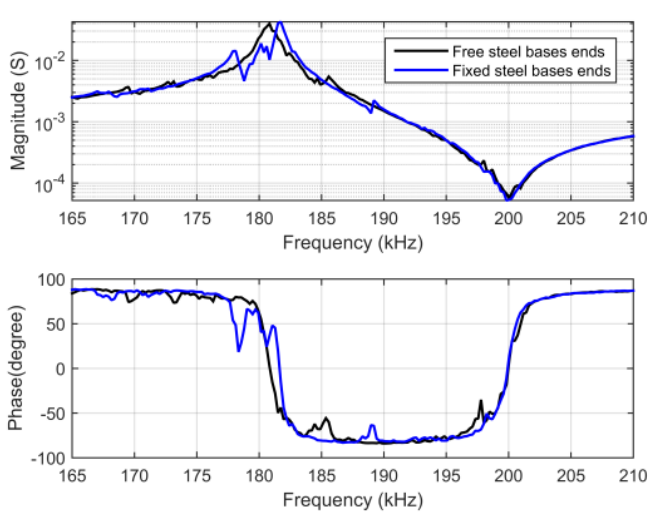

Figure. 17: Electric admittance spectrum of the piezoelectric specimen with free and fixed boundary conditions at the steel bases ends

The simulation results in Figure. 17 show that the model is able to reproduce the parasitic vibration modes, their frequencies and magnitudes depending on the boundary condition at the ends of the steel bases. In fact, the real boundary conditions are unknown and are situated between free-moving and fixed. According to the model assumptions, the components in contact with the piezoelectric specimen are responsible for the appearance of these modes. To investigate the assumption that the variation of the contact interfaces between the components of the structure with the applied stress are due to the surfaces roughness, we varied the equivalent stiffness $k_{E}$ at the contact interfaces.

It can clearly be seen in Figure 18 that the contact interface with the presence of the auxiliary electrodes could contribute to the shift of the resonance and the anti-resonance frequencies to higher values and the increase of the parasitic vibration modes. Furthermore, the model shows that the material and the geometry of the components of the structure play a key role in the effect of the contact interfaces.
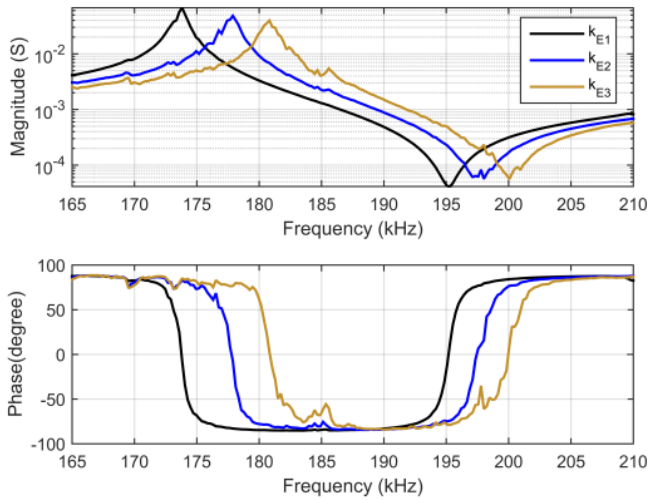

Figure. 18: Effect of the equivalent stiffness on the electrical admittance spectrum $k_{E 3}>k_{E 2}>k_{E 1}$

Figure. 19 shows the simulation results for two tests, first with another material for the bases (Aluminum) and 
second with cylindrical bases (radius $=60 \mathrm{~mm}$ ).
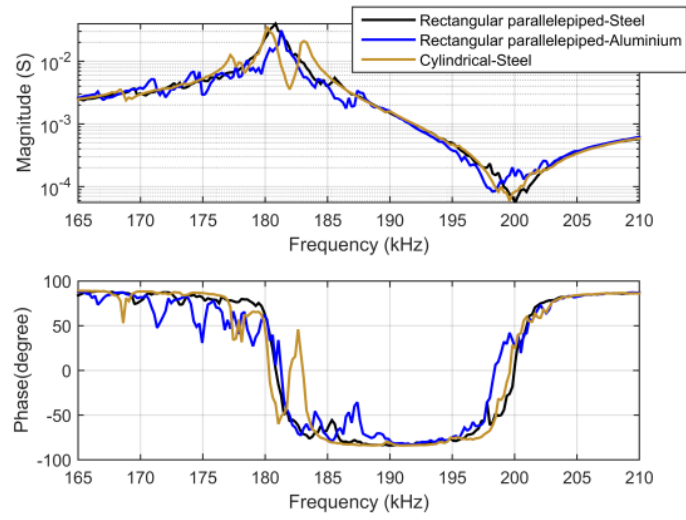

Figure. 19: Electric admittance spectrum of the piezoelectric specimen with different shape and material of the bases

Based on this analysis, we can conclude that the electrical admittance spectrum of the piezoelectric specimen clearly depends on: the components of the structure (the geometry and the material) and the contact interface between them. These are the key elements to take into consideration in order to enhance the quality of the spectrum.

\section{Enhancement of the resonant piezoelectric structure}

The goal of this section is to investigate a solution in order to minimize the parasitic vibration modes that degrade the quality of the main vibration mode (reducing the force measuring range). According to the results of the analysis in the previous section, to minimize the parasitic vibration modes the piezoelectric specimen should not be highly constrained at the contact interfaces and should be decoupled from the rest of the structure in order not to excite the natural vibration modes of the structure through the propagation of the acoustic wave. The proposed solution is to introduce a layer made of soft material (characterized by a low Young modulus). The simulation results in Figure. 20 show that the added soft material significantly reduces the parasitic modes and that the resonance frequency approaches the resonance seen in free vibration conditions. Based on the simulation results we setup an experimental test to verify the effectiveness of the soft material based layer. We tested two materials: rubber and Polytetrafluoroethylene (PTFE). The tested thickness of the materials were selected based on their immediate availability in our laboratory.
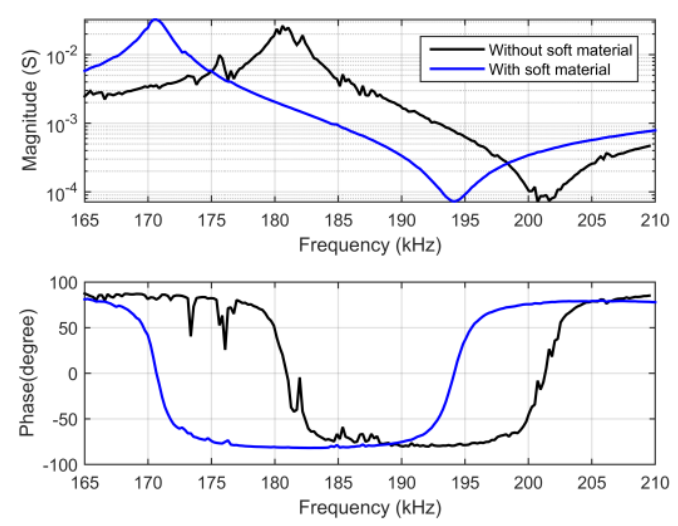

Figure. 20: Effect of the soft material layer on the electrical admittance spectrum

In the first test, the piezoelectric specimen was sandwiched between two layers of $2 \mathrm{~mm}$ thick rubber. The measured electrical admittance spectrum of the second vibration mode in Figure. 21.a shows that the parasitic modes are reduced; however, the sensitivity of the sensor was degraded.
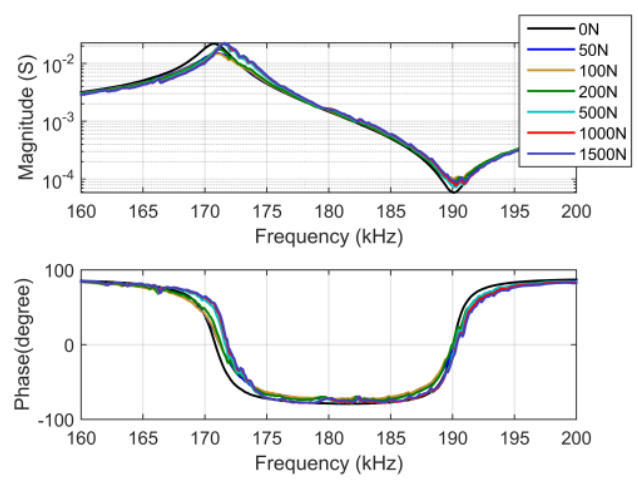

(a)

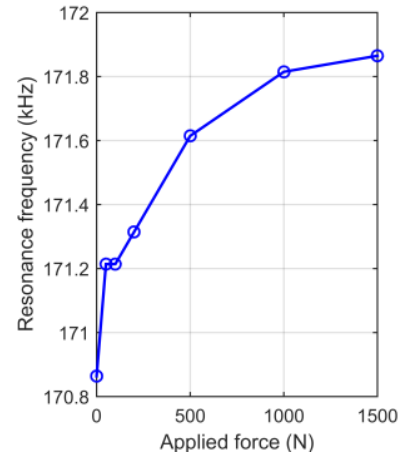

(b)

Figure. 21: (a) Measured electrical admittance spectrum for the second vibration mode when adding the rubber layers and (b) the resonance frequency shift

In the second test, two layers of $3 \mathrm{~mm}$ thick PTFE were used. The measured electrical admittance spectrum in Figure 22.a shows that the parasitic modes are eliminated allowing a measuring range up to $1500 \mathrm{~N}$ with maximal sensitivity of $1.9 \mathrm{~Hz} / \mathrm{N}$. The quality factor seems to decrease drastically in comparison to the 
response of the PTFE-less structure; this could be explained by high mechanical losses on the PTFE layer. The results show that the soft material layer must be well designed to a achieve good performance of the transducer.
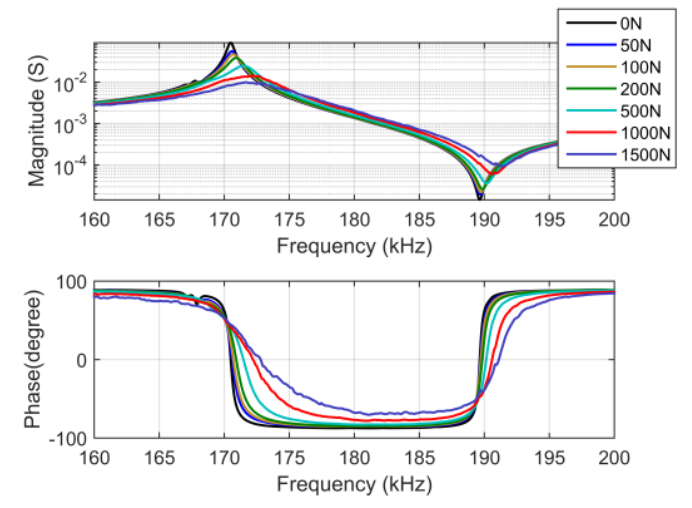

(a)

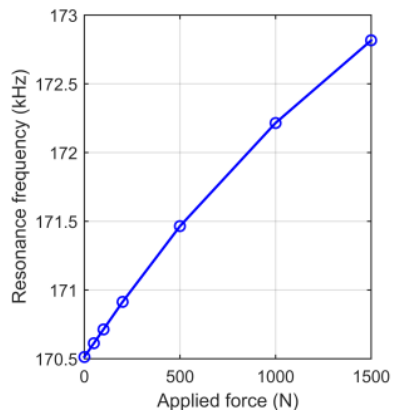

(b)

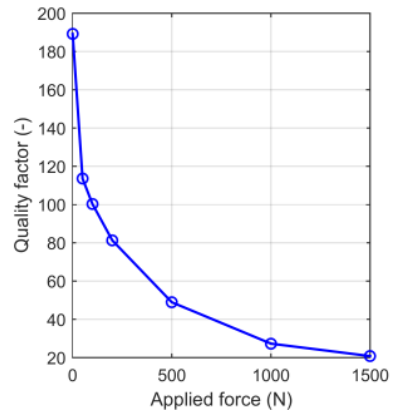

(c)
Figure. 22: (a) Measured electrical admittance spectrum for the second vibration mode when adding the PTFE layers with (b) the resonance frequency and (c) the quality factor as function of the applied force

\section{Conclusion}

A resonant bulk acoustic wave piezoelectric transducer for high static force sensing was investigated in this paper. The preliminary experimental results based on the electrical admittance spectrum measurements highlight the sensitivity of the piezoelectric specimen's in response to the applied mechanical force. However, parasitic modes appear on the spectrum of the main vibration mode when increasing the applied force, therefore the purity of the response is decreased, leading to the loss of a clear signature of the stress that can be detected by electronics. The analysis based on the developed analytical model show that the stress dependence of the electrical admittance spectrum is due to the variation of the effective parameters of the piezoelectric material with respect to the applied stress. Those variations of the effective parameters of the piezoelectric material with respect to the applied force were successfully predicted by an in-house developed multi-scale model. The finite element analysis shows that the components of the structure and the boundary condition at the contact interface impact considerably the electrical admittance spectrum. A solution was then proposed to enhance the performance of the transducer by adding a soft material layer (based on PTFE material). Good results were obtained in terms of the increase of the force measuring range. Further experimental and modeling work is ongoing to achieve a robust transducer based on the Bragg reflector technique that will be capable of decoupling the piezoelectric specimen from the rest of the structure with minimum elastic losses.

\section{References}

[1] E. K. J. J. FradenTichý, J.Erhart, Fundamentals of Piezoelectric Sensorics, S. B. Heidelberg, Ed. Springer Berlin Heidelberg, 2010.

[2] S. Ying, W. Zhichen, and D. Yanliang, "Theoretical and experimental research on piezoelectric sensors response to dynamic strain," in Electronic Measurement and Instruments, 2007. ICEMI '07. 8th International Conference on, Aug 2007, pp. 4-194-4-198.

[3] J. F. Tressler, S. Alkoy, and R. E. Newnham, "Piezoelectric sensors and sensor materials," Journal of Electroceramics, vol. 2, no. 4, pp. 257272, 1998. [Online]. Available: http://dx.doi.org/10.1023/A:1009926623551

[4] J. Fraden, Handbook of Modern Sensors, 4th ed. Springer New York, 2010.

[5] J.Yong, "Contribution à l'étude d'un capteur de force piézoélectrique résonant à forte raideur pour interfaces haptiques aéronautiques," Ph.D. dissertation, University Paul Sabatier - Toulouse III, 2013.

[6] M. Lord and D. M. Smith, "Static response of a simple piezoelectric load cell," Journal of Biomedical Engineering, vol. 5, no. 2, pp. 162 164, 1983. [Online]. Available: http://www.sciencedirect.com/science/article/pii/0141542583900377

[7] O. Mack, "Investigations of piezoelectric force measuring devices for use in legal weighing metrology," Measurement, vol. 40, no. 7â€"8, pp. 746 - 753, 2007, precision Measurement of Force, Mass, and Torque. [Online]. Available: http://www.sciencedirect.com/science/article/pii/S026322410600073X

[8] D. M. Stefanescu, Handbook of Force Transducers, S. B. Heidelberg, Ed. Springer-Verlag Berlin Heidelberg, 2011, no. 612.

[9] A. Arnau, T. Sogorb, and Y. Jimenez, "A new method for continuous monitoring of series resonance frequency and simple determination of motional impedance parameters for loaded quartz-crystal resonators," IEEE Transactions on Ultrasonics, Ferroelectrics, and Frequency Control, vol. 48, no. 2, pp. 617-623, March 2001.

[10] C. Barthod, Y. Teisseyre, C. GÃ@hin, and G. Gautier, "Resonant force sensor using a $\{\mathrm{PLL}\}$ electronic," Sensors and Actuators A: Physical, vol. 104, no. 2, pp. 143 - 150, 2003. [Online]. Available: http://www.sciencedirect.com/science/article/pii/S0924424703000050

[11] J. Sell, A. Niedermayer, and B. Jakoby, "Digital phase-locked loop circuit for driving resonant sensors," Procedia Engineering, vol. 5, pp. $204-207,2010$, eurosensor $\{$ XXIV $\}$ ConferenceEurosensor $\{$ XXIV $\}$ Conference. [Online]. Available: http://www.sciencedirect.com/science/article/pii/S1877705810006302

[12] E. Benes, M. Gröschl, W. Burger, and M. Schmid, "Sensors based on piezoelectric resonators," Sensors and Actuators A: Physical, vol. 48, no. 1, pp. $1-21,1995$. [Online]. Available: http://www.sciencedirect.com/science/article/pii/0924424795008462

[13] B. Drafts, "Acoustic wave technology sensors," Microwave Theory and Techniques, IEEE Transactions on, vol. 49, no. 4, pp. 795-802, Apr 2001.

[14] M. J. Vellekoop, "Acoustic wave sensors and their technology," Ultrasonics, vol. 36, no. 1, pp. 7 - 14, 1998, ultrasonics International 1997. [Online]. Available: http://www.sciencedirect.com/science/article/pii/S0041624X97001467

[15] M. Olfatnia, T. Xu, J. Miao, L. Ong, X. Jing, and L. Norford, "Piezoelectric circular microdiaphragm based pressure sensors," Sensors and Actuators A: Physical, vol. 163, no. 1, pp. 32 - 36, 2010. [Online]. Available: http://www.sciencedirect.com/science/article/pii/S0924424710002785 
[16] V. Mohammadi, M. H. Sheikhi, S. Torkian, A. Barzegar, E. Masumi, and S. Mohammadi, "Design, modeling and optimization of a piezoelectric pressure sensor based on thin-film pzt diaphragm contain of nanocrystalline powders," in Mechatronics and its Applications, 2009. ISMA '09. 6th International Symposium on, March 2009, pp. 1-7.

[17] T. Shanmuganantham, U. J. Gogoi, and J. Gandhimohan, "A study scheme of energy harvesting process of mems piezoelectric pressure sensor," in 2016 International Conference on Circuit, Power and Computing Technologies (ICCPCT), March 2016, pp. 1-5.

[18] C. W. Tan, A. G. P. Kottapalli, Z. H. Wang, X. Ji, J. M. Miao, G. Barbastathis, and M. Triantafyllou, "Damping characteristics of a micromachined piezoelectric diaphragm-based pressure sensor for underwater applications," in 2011 16th International Solid-State Sensors, Actuators and Microsystems Conference, June 2011, pp. 7275.

[19] L. Ngalamou, N. Noury, E. Chamberod, and P. Benech, "Analysis of the sensitivity and the temperature influence of a static force sensor based on a pvdf resonator," Sensors and Actuators A: Physical, vol. 57, no. 3, pp. 173 - 177, 1996. [Online]. Available: http://www.sciencedirect.com/science/article/pii/S0924424797801100

[20] S. Bah, G. Quezel, P. Benech, L. Ngalamou, and J. F. Legrand, "Effect of compressive stress on a composite resonator based on pvf 2," in Ultrasonics Symposium, 1992. Proceedings., IEEE 1992, Oct 1992, pp. 973-975 vol.2.

[21] D. Shmilovitz, M. Kerman, S. Ozeri, and S. Engelberg, "Static force measurement with piezoelectric sensors based on pseudorandom sequences," in Industrial Electronics, 2008. IECON 2008. 34th Annual Conference of IEEE, Nov 2008, pp. 1794-1797.

[22] S. Ozeri and D. Shmilovitz, "Static force measurement by piezoelectric sensors," in 2006 IEEE International Symposium on Circuits and Systems, May 2006, pp. 4 pp.-.

[23] Q. Zhang and J.Zhao, "Electromeasuremetal properties of lead zirconate titanate piezoceramics under the influence of mechanical stresses," Ultrasonics, Ferroelectrics, and Frequency Control, IEEE Transactions on, vol. 46, no. 6, pp. 1518-1526, Nov 1999.

[24] S. Lin, "Study on the radial vibration of a piezoelectric ceramic thin ring with an inner metal disc," Journal of Physics D: Applied Physics, vol. 39, no. 21, p. 4673, 2006. [Online]. Available: http://stacks.iop.org $/ 0022-3727 / 39 / \mathrm{i}=21 / \mathrm{a}=026$

[25] A. Iula, N. Lamberti, and M. Pappalardo, "A model for the theoretical characterization of thin piezoceramic rings," IEEE Transactions on Ultrasonics, Ferroelectrics, and Frequency Control, vol. 43, no. 3, pp. 370-375, May 1996. 